Irish Math. Soc. Bulletin

Number 80, Winter 2017, 21-33

ISSN 0791-5578

\title{
Generalised Hopficity and Products of the Integers
}

\author{
BRENDAN GOLDSMITH \\ In Memoriam: Eoin Coleman (Oren Kolman) 1959-2015.
}

\begin{abstract}
Hopfian groups have been a topic of interest in algebraic settings for many years. In this work a natural generalization of the notion, so-called R-Hopficity is introduced. Basic properties of R-Hopfian groups are developed and the question of whether or not infinite direct products of copies of the integers are R-Hopfian is considered. An unexpected result is that the answer to this purely algebraic question depends on the set theory assumed.
\end{abstract}

\section{INTRODUCTION}

The finiteness of a set $S$ can be expressed in two equivalent ways in terms of functions from $S \rightarrow S$. Thus the set $S$ is finite if, and only if, every one-one function $S \rightarrow S$ is invertible, if, and only if, every onto function $S \rightarrow S$ is invertible. The comparable statements in the category of all groups $\mathcal{G}$ fail to be true: multiplication by the prime $p$ in the additive group of integers is a one-one homomorphism (or monomorphism) which is not an invertible homomorphism (or automorphism) and the same multiplication in the quasi-cyclic group $\mathbb{Z}\left(p^{\infty}\right)$ is an onto homomorphism (or epimorphism) which is not an automorphism. Nonetheless these statements about homomorphisms may be used to define certain classes of groups which will contain all finite groups. Specifically we shall say that a (possibly non-commutative) group $G$ is

(i) co-Hopfian if every monic endomorphism of $G$ is an automorphism;

(ii) Hopfian if every epic endomorphism of $G$ is an automorphism.

The terminologies arise from the fact that groups satisfying condition (ii) arose in work of the topologist H. Hopf on fundamental groups of closed two-dimensional orientable surfaces, while (i) is in

2010 Mathematics Subject Classification. 20K30, 20k20.

Key words and phrases. Hopficity, R-Hopficity, Baer-Specker group.

Received on 19-1-2017; revised 4-7-2017. 
a certain weak sense a notion dual to (ii). Co-Hopfian and Hopfian groups were investigated under the names $S$-groups and $Q$-groups respectively by Baer in [1]. The terminology using $S, Q$ reflects the fact that it is easy to establish that (i) and (ii) are respectively equivalent to the following:

(S) $G$ cannot have a proper isomorphic subgroup;

(Q) $G$ cannot have a proper isomorphic quotient group.

There is an extensive literature on both Hopfian and co-Hopfian groups, the papers [1, 2, 3, 5, 9, 10, 12, 13, 16, 17] and the references therein give a small cross-section of the literature.

To simplify our presentation we are going to consider only additively written Abelian groups but many of our results can be extended without difficulty to both the category of (all) groups and the category of modules over a commutative ring. Furthermore, we will focus attention on a weaker version of Hopficity introduced below, even though there are many results of a dual nature that can be established for the corresponding generalisation of co-Hopficity.

If one interprets the defining condition (ii) of Hopficity as saying that a group $G$ is Hopfian if every surjective endomorphism has a two-sided inverse, then it is possible to weaken the definition in two natural ways, where in an obvious notation the letters ' $R$, $L$ ' stand for 'right' and 'left' respectively. Note that in this paper, maps are always written on the left.

Definition 1.1. A group $G$ is said to be R-Hopfian [L-Hopfian] if for every surjection $\phi \in \operatorname{End}(G)$, there is an endomorphism $\psi$ of $G$ such that $\phi \psi=1_{G}\left[\psi \phi=1_{G}\right]$.

Observe firstly that if $G$ is Hopfian, then certainly $G$ is both RHopfian and L-Hopfian. Moreover, if $G$ is L-Hopfian and $\phi$ is a surjection, then the equation $\psi \phi=1_{G}$ implies that $\phi$ is also an injection, so that $\phi$ is an automorphism of $G$. Consequently the class of L-Hopfian groups coincides with the class of Hopfian groups. Our focus will therefore be on the class of R-Hopfian groups.

The paper is organised into a further three sections: in Section 2 we will explore some elementary properties of our new class and how it relates to the original concept of Hopficity. The rather simple Example 2.4 is important for subsequent developments because it is well known - see [18] or [7, Lemma 112.1] - that a group has regular endomorphism ring if, and only if, it is both (Ker)-direct and (Im)Direct in the sense that the kernel (image) of every endomorphism 
is a direct summand. Recently the notions of (Ker)-directness and (Im)-directness of modules have been investigated in [14, 15], where the concepts are called Rickart modules and dual Rickart modules; a weakening of the idea of (Ker)-directness will be key to understanding R-Hopficity.

In the third section we investigate the behaviour of the class under the formation of direct sums. This is a natural consideration in light of the rather surprising examples given by Corner [5] in 1965:

(C1) the direct sum of two torsion-free Hopfian groups need not be Hopfian

(C2) there exist torsion-free Hopfian groups $A$ such the direct sum $A \oplus A$ is not Hopfian.

We shall show that a result analogous to (C1) holds for R-Hopfian groups but the analogue of $(\mathrm{C} 2)$ seems, on the surface at least, to be extremely difficult.

Sections 2 and 3 are reasonably straightforward and do not require a great deal of specialised knowledge.

The final section on the Baer-Specker groups, i.e., direct products of the group of integers, $P_{\kappa}=\mathbb{Z}^{\kappa}$, makes use of much deeper results and introduces a number of ideas relating to the interaction of set theory with group theory. The main result of that section is the rather surprising fact that the seemingly totally algebraic notion of R-Hopficity leads one to natural questions whose answers are independent of the usual Zermelo-Fraenkel axioms of set theory along with the axiom of choice, ZFC.

We finish off this introduction by noting that notation in the paper is standard as in the two volumes of Fuchs [7, 8]; in particular mapping are consistently written on the left and for an Abelian group $G$, the ring of endomorphisms of $G$ shall be denoted by $\operatorname{End}(G)$ and the group of all homomorphisms: $A \rightarrow B$ shall be denoted $\operatorname{Hom}(A, B)$. We shall frequently make use of the well-known representation of endomorphisms of the direct sum of groups $A, B$ by a matrix: if $\psi$ is an endomorphism of $A \oplus B$, then $\psi$ has a matrix representations of the form $\Delta=\left(\begin{array}{ll}\alpha & \gamma \\ \delta & \beta\end{array}\right)$ where $\alpha \in \operatorname{End}(A), \beta \in \operatorname{End}(B), \gamma \in \operatorname{Hom}(B, A)$ and $\delta \in \operatorname{Hom}(A, B)$. The additive group of integers is denoted by $\mathbb{Z}$, while the additive group of rationals is denoted by $\mathbb{Q}$.

All groups will be additively written Abelian groups. 


\section{Elementary Results}

The notion of direct finiteness provides the connection between Hopficity and R-Hopficity. Recall that a group $G$ is said to be directly finite if, given endomorphisms $\phi, \psi$ of $G$ with $\phi \psi=1_{G}$, the identity map on $G$, then $\psi \phi=1_{G}$ also.

It is easy to show for an Abelian group $G$, that direct finiteness is equivalent to the statement that $G$ cannot have a proper isomorphic direct summand.

Proposition 2.1. A group $G$ is Hopfian if, and only if, it is RHopfian and directly finite; in particular, if $\operatorname{End}(G)$ is commutative, then $G$ is R-Hopfian if, and only if, it is Hopfian.

Proof. If $G$ is Hopfian then every surjection has an inverse, so $G$ is certainly R-Hopfian. However, if $\alpha \beta=1_{G}$ for some $\alpha, \beta \in \operatorname{End}(G)$, then $\alpha$ is surjective and so, by the Hopficity of $G$, it has an inverse $\alpha^{-1}$. It follows immediately that $\beta=\alpha^{-1}$ and so $\beta \alpha=1_{G}$, whence $G$ is directly finite.

Conversely, given any surjection $\phi \in \operatorname{End}(G)$, R-Hopficity ensures the existence of an endomorphism $\psi$ such that $\phi \psi=1_{G}$. By direct finiteness, we have that $\psi \phi$ is also equal to $1_{G}$ and so $\phi$ is invertible with inverse $\psi$. Since $\phi$ was arbitrary, we have that $G$ is Hopfian.

The particular case in which $\operatorname{End}(G)$ is commutative is now immediate.

Corollary 2.2. An indecomposable group is Hopfian if, and only if, it is $R$-Hopfian. In particular, the group $\mathbb{Z}\left(p^{\infty}\right)$ is not $R$-Hopfian for any prime $p$.

Proof. The necessity is immediate and doesn't require the indecomposability. Conversely suppose that $G$ is R-Hopfian. It suffices by Proposition 2.1 to show that $G$ is directly finite. Suppose then that $f g=1_{G}$ for endomorphisms $f, g$ of $G$. Then $g f$ is an idempotent endomorphism which cannot be the zero map and so the indecomposability of $G$ forces $g f=1_{G}$, as required.

Given the close connection between Hopfian and R-Hopfian groups just established, we would expect R-Hopfian groups to share some properties known for Hopfian groups. Our first result is an analogue of such a property of Hopfian groups.

Proposition 2.3. A direct summand of an $R$-Hopfian group $G$ is again R-Hopfian. 
Proof. Suppose then that $G=H \oplus S$ and let $\alpha$ be an arbitrary surjection in $\operatorname{End}(H)$. Then $\psi=\alpha \oplus 1_{S}$ is a surjection in $\operatorname{End}(G)$ and so there is a $\phi \in \operatorname{End}(G)$ such that $\psi \phi=1_{G}$. Using the standard matrix representation of endomorphisms of a direct sum, this means that

$$
\left(\begin{array}{cc}
\alpha & 0 \\
0 & 1_{S}
\end{array}\right) \cdot\left(\begin{array}{cc}
\mu & \nu \\
\rho & \sigma
\end{array}\right)=\left(\begin{array}{cc}
1_{H} & 0 \\
0 & 1_{S}
\end{array}\right), \quad \text { where } \phi=\left(\begin{array}{cc}
\mu & \nu \\
\rho & \sigma
\end{array}\right) .
$$

Thus $\alpha \mu=1_{H}$, and so, since $\alpha$ was an arbitrary surjection in $\operatorname{End}(H), H$ is R-Hopfian.

Recall that a ring $R$ is said to be (von Neumann) regular if, given any $a \in R$ there exists a $b \in R$ such that $a b a=a$. We can then deduce a simple result which will provide some motivation for a somewhat deeper result that gives us a classification of R-Hopfian groups.

Example 2.4. If $G$ is a group with a regular endomorphism ring, then $G$ is R-Hopfian.

Proof. Suppose that $\alpha$ is an arbitrary surjection in $\operatorname{End}(G)$, then by regularity, there is a $\beta \in \operatorname{End}(G)$ such that $\alpha \beta \alpha=\alpha$. If $x \in G$, then $x=\alpha(y)$ for some $y \in G$ and so

$$
x=\alpha(y)=\alpha \beta \alpha(y)=\alpha \beta(x) \text { for all } x \in G .
$$

Hence the composition $\alpha \beta$ is the identity $1_{G}$ on $G$ and $G$ is RHopfian.

Note that it follows easily from Example 2.4 that the class of RHopfian groups properly contains the class of Hopfian groups: it is well known that the ring of linear transformations of an infinite dimensional rational vector space is regular and hence, for example, the group $G=\bigoplus_{\aleph_{0}} \mathbb{Q}$ is R-Hopfian but it is clearly not Hopfian since it contains a proper isomorphic direct summand.

As mentioned in the Introduction, a group with regular endomorphism ring has the property that the kernel of every endomorphism is a direct summand of the group; the group is then said to be (Ker)-direct. A natural weakening of this concept is the following:

Definition 2.5. A group $G$ is said to be (sKer)-direct if the kernel of each surjective endomorphism of $G$ is a direct summand of $G$.

The proof of the next result is reasonably well known but we give a detailed proof for completeness. 
Theorem 2.6. A group $G$ is R-Hopfian if, and only if, it is (sKer)direct.

Proof. Assume that $G$ is R-Hopfian and that $\phi$ is an arbitrary surjective endomorphism of $G$. Then there exists a (necessarily monic) endomorphism $\alpha$ such that $\phi \alpha=1_{G}$. Since $\alpha \phi$ is then an idempotent endomorphism of $G$, its kernel is a summand of $G$. However, the fact that $\alpha$ is monic implies that $\operatorname{Ker} \phi=\operatorname{Ker} \alpha \phi$ and so $\operatorname{Ker} \phi$ is a summand of $G$. Since $\phi$ was arbitrary, we have that $G$ is (sKer)direct.

Conversely suppose that $G$ is (sKer)-direct and let $\sigma$ be an arbitrary surjective endomorphism of $G$. Then $G=\operatorname{Ker}(\sigma) \oplus T$ for some complement $T$. Now $\sigma \uparrow T: T \rightarrow G$ has the property that $\sigma \uparrow T(T)=G$ since the surjectivity of $\sigma$ means that an arbitrary $x \in G$ has the form $x=\sigma(y)$ for some $y \in G$; but $y=k+t$ for some $k \in \operatorname{Ker}(\sigma), t \in T$ and so $x=\sigma(k+t)=\sigma(t)$. Since $\operatorname{Ker}(\sigma\lceil T)=T \cap \operatorname{Ker}(\sigma)=\{0\}$, we conclude that $\sigma\lceil T$ is an isomorphism $T \rightarrow G$. So there is an endomorphism $\eta: G \rightarrow T$ such that $\left(\sigma\lceil T) \eta=1_{G}\right.$. Hence $\sigma \eta=1_{G}$ and $G$ is R-Hopfian.

It is clear from Theorem 2.6 that the class of R-Hopfian groups is large: free and divisible torsion-free groups of arbitrary rank, elementary $p$-groups of arbitrary dimension and torsion-free reduced algebraically compact groups are all R-Hopfian. (The first three classes are easy to see while the final one results from the fact that in this class, the kernel of any endomorphism is both complete and pure and hence a summand - see, for example [7, Corollary 39.3].)

Further results including a detailed discussion of R-Hopficity in the context of Abelian p-groups, may be found in [11].

\section{Direct Sums}

Our first result, which has been been proved in an outline form in [11, Proposition 3.5], is the simple:

Proposition 3.1. If $A$ is an $R$-Hopfian group, $B$ a Hopfian group and

$\operatorname{Hom}(A, B)=0$, then $A \oplus B$ is $R$-Hopfian.

Proof. In the standard matrix representation of an endomorphism of $G=A \oplus B$, the entry in the $(2,1)$ position must be 0 since $\operatorname{Hom}(A, B)=0$. Let $\Delta=\left(\begin{array}{cc}\mu & \nu \\ 0 & \sigma\end{array}\right)$ be an arbitrary surjection and observe that this forces $\sigma$ to be a surjection of $B$. Since $B$ is Hopfian 
this implies that $\sigma$ is an automorphism of $B$. We claim that $\mu$ is also a surjection. To see this pre-multiply $\Delta$ by the invertible matrix $\left(\begin{array}{cc}1 & -\nu \sigma^{-1} \\ 0 & \sigma^{-1}\end{array}\right)$ which corresponds to performing two standard elementary row operations in the normal diagonalizing process, so that we again obtain a surjection which is equal to the diagonal matrix $\left(\begin{array}{ll}\mu & 0 \\ 0 & 1\end{array}\right)$; consequently $\mu$ is a surjection as claimed and since $A$ is R-Hopfian, there is an endomorphism $\alpha$ of $A$ with $\mu \alpha=1_{A}$. The proof is completed by observing that post-multiplying $\Delta$ with the matrix $\left(\begin{array}{cc}\alpha & -\alpha \nu \sigma^{-1} \\ 0 & \sigma^{-1}\end{array}\right)$ this is the standard technique for inverting a matrix but here we are producing just a right inverse - yields the identity matrix.

We now show that Proposition 3.1 fails if we replace the condition $\operatorname{Hom}(A, B)=0$ with $\operatorname{Hom}(B, A)=0$. First we need a technical lemma.

Lemma 3.2. Let $A$ be a group having a surjection $\alpha$ whose kernel is a summand of $A$ and suppose that there is a surjection $\gamma$ from Ker $\alpha$ onto the group $B$. Then there is a mapping $\delta: A \rightarrow B$ extending $\gamma$ such that the group $A \oplus B$ has a surjection with matrix representation $\Delta=\left(\begin{array}{ll}\alpha & 0 \\ \delta & 0\end{array}\right)$.

Proof. Let $A=K \oplus A_{1}$ where $K=$ Ker $\alpha$ and define the mapping $\delta: A \rightarrow B$ by

$$
\delta(z)=\left\{\begin{array}{rll}
\gamma(z) & : & z \in K \\
0 & : & z \in A_{1}
\end{array}\right.
$$

. Now let $b$ be an arbitrary element of $B$. Then there is an element $x \in K$ such that $\delta(x)=\gamma(x)=b$. Direct computation shows that $\Delta$ maps the element $(x, 0)$ of $A \oplus B$ onto $(0, b)$. Furthermore, if $a$ is an arbitrary element of $A$, then there is an element $y \in A$ with $\alpha(y)=a$; note that we may assume that $y \in A_{1}$. Again direct computation, noting that $\delta(y)=0$ since $y \in A_{1}$, gives us that $\Delta$ maps $(y, 0)$ onto $(a, 0)$. It follows that $\Delta$ is a surjection of $A \oplus B$, as claimed.

Theorem 3.3. If $A$ is free of infinite rank $\kappa$ and $B$ is a group of cardinality $\leq \kappa$, then the group $G=A \oplus B$ is R-Hopfian if, and only if, $B$ (or equivalently $G$ ) is free.

Proof. The sufficiency is clear: free groups are always R-Hopfian.

For the necessity, decompose $A$ as $A_{1} \oplus A_{2}$ with $A \cong A_{1} \cong A_{2}$ and define $\alpha$ to map $A_{1}$ isomorphically onto $A$ and to act as 0 
on $A_{2} ; \alpha$ is then a surjection of $A$ having kernel isomorphic to $A$. Furthermore, since Ker $\alpha$ is free of rank $\kappa$, there is a surjection $\gamma$ : Ker $\alpha \rightarrow B$. Applying Lemma 3.2, we obtain a surjection $\Delta$ of $A \oplus B$ with matrix representation $\left(\begin{array}{ll}\alpha & 0 \\ \delta & 0\end{array}\right)$ for some $\delta: A \rightarrow B$ which extends $\gamma$. Now every endomorphism of $A \oplus B$ has a matrix representation of the form $\left(\begin{array}{ll}\mu & \nu \\ \rho & \sigma\end{array}\right)$ and so if $G$ is R-Hopfian, there is a product $\left(\begin{array}{ll}\alpha & 0 \\ \delta & 0\end{array}\right) \cdot\left(\begin{array}{ll}\mu & \nu \\ \rho & \sigma\end{array}\right)$ equal to the identity matrix for some choice of $\mu, \nu, \rho, \sigma$.

Hence we deduce that $\alpha \nu=0$ and $\delta \nu=1_{B}$; it follows immediately from the latter equality that Ker $\nu=0$. Furthermore, the first equality forces $\nu(B) \leq \operatorname{Ker} \alpha$, so that $\nu(B)$ is free, being a subgroup of the free group $A$. Since $\nu$ has trivial kernel, we have that $B \cong \nu(B)$, so that $B$ is necessarily free.

The choice of $B=\mathbb{Q}$ in the above theorem yields the desired analogue of Corner's example (C1): the direct sum of two R-Hopfian groups need not be R-Hopfian. We record this as:

Corollary 3.4. The direct sum of two $R$-Hopfian groups need not be R-Hopfian, even when one of the groups is Hopfian.

Corollary 3.5. If $A$ is free of rank $\kappa$ and $B$ has a non-free summand $X$ of cardinality $\leq \kappa$, the group $A \oplus B$ is not $R$-Hopfian. In particular, an $R$-Hopfian group having a free summand of infinite rank is necessarily torsion-free.

Proof. Suppose that $X$ is a summand of $B$ and $|X| \leq \kappa$. If $A \oplus B$ were R-Hopfian, then $A \oplus X$ would also be R-Hopfian, contrary to Theorem 3.3. In particular, if $G$ is R-Hopfian of the form $G=F \oplus H$, where $F$ is free of infinite rank, then $H$ must be torsion-free since otherwise $G$ would have an R-Hopfian summand of the form $F \oplus C$, where $C$ is either finite or of the form $\mathbb{Z}\left(p^{\infty}\right)$ for some prime $p$, both of which are impossible.

Note a consequence of the above result: unlike the situation for Hopfian groups where the direct sum of a Hopfian group and a cyclic group is necessarily Hopfian (see [13] or [9]), the direct sum of a free (and hence R-Hopfian) group of infinite rank and a finite cyclic group is never R-Hopfian. In fact, this example shows that the class 
of R-Hopfian groups does not satisfy the weak closure property of Hopfian groups (see, for example, [10, Proposition 2.3]): if $0 \rightarrow$ $H \rightarrow G \rightarrow K \rightarrow 0$ is an exact sequence and $H, K$ are both Hopfian and if $H$ is left invariant by each surjection $\phi: G \rightarrow G$, then $G$ is Hopfian. In particular, extensions of Hopfian torsion groups by torsion-free Hopfian groups are again Hopfian but this is no longer true if we replace Hopfian by R-Hopfian.

If we apply our arguments to countable groups we can say a little more:

Theorem 3.6. A countable group $G$ is $R$-Hopfian if, and only if, $G$ is free or $G$ has the form $G=F \oplus N$, where $F$ is free of finite rank and $N$ is $R$-Hopfian with $\operatorname{Hom}(N, \mathbb{Z})=0$.

Proof. The sufficiency is straightforward: if $G$ is free then it is (Ker)direct and hence R-Hopfian, while if it has the given form $G=F \oplus N$, a direct application of Proposition 3.1 (i) shows that $G$ is R-Hopfian. Conversely, suppose that $G$ is R-Hopfian and not free. Since $G$ is countable, we may make use of a standard result on countable Abelian groups due to Stein - see, for example, [7, Corollary 19.3] that $G=F \oplus N$, where $F$ is free and $\operatorname{Hom}(N, \mathbb{Z})=0$; note that $N$ is also R-Hopfian as a summand of an R-Hopfian group. It remains only to show that $F$ has finite rank. However, if $F$ were of infinite rank, then it would follow from Theorem 3.3 that $G$ is not R-Hopfian - contradiction.

Corollary 3.7. An R-Hopfian group $G$ which is not reduced is of the form $G=\mathbb{Q}^{(\kappa)} \oplus X$, where $\kappa \neq 0$ is a cardinal, $X$ is reduced $R$-Hopfian and $X$ does not have a free summand of infinite rank.

Proof. As $G$ is not reduced it is of the form $G=D \oplus X$, where $D$ is divisible and $X$ is a reduced R-Hopfian group. Since for all primes $p$, the group $\mathbb{Z}\left(p^{\infty}\right)$ is directly finite but not Hopfian, it is not R-Hopfian. Thus $D$ must be torsion-free divisible, $D=\mathbb{Q}^{(\kappa)}$ for some cardinal $\kappa \neq 0$. However, if $X$ has a free summand $F$ of infinite rank, then $G$ has a summand (necessarily R-Hopfian) of the form $\mathbb{Q} \oplus F$, contrary to Theorem 3.3 . Thus $X$ does not have a free summand of infinite rank.

The requirement in Theorem 3.3 that $|B| \leq|A|$ cannot be omitted. The proof of the following result is based on modern realization theorems which utilise sophisticated arguments deriving from the 
combinatorial arguments of Shelah's Black Box. We state it without proof.

Theorem 3.8. For each infinite cardinal $\kappa$ there is a non-free torsionfree $R$-Hopfian group $G$ having a free summand of rank $\kappa$.

We finish this section by posing the analogue of Corner's result $(\mathrm{C} 2)$ :

Problem: Does there exist an R-Hopfian group $A$ such that the square $A \oplus A$ is not R-Hopfian?

We remark that it is not easy to just adapt Corner's original argument since the key point in his solution is that the group $A \oplus A$ is not directly finite and hence not Hopfian. However, failure to be directly finite does not imply failure to be R-Hopfian.

\section{BAer-Specker Groups}

If $\kappa$ is an infinite cardinal then the groups $P_{\kappa}=\mathbb{Z}^{\kappa}$ are usually referred to as higher Baer-Specker groups; when $\kappa=\aleph_{0}$ we normally write $P$ rather than $P_{\aleph_{0}}$ and $P$ is the familiar Baer-Specker group. (For an informative introduction with an excellent list of references, see the article by Eoin Coleman in the IMS Bulletin [4].) In this section we investigate the groups $P_{\kappa}$ in relation to the property of being R-Hopfian. Many properties of such products derive from fundamental work of Nunke but we shall find the more modern exposition in Eklof-Mekkler [6, Chapter IX] more useful for our purposes; in particular [6] contains the necessary set-theoretic background required and a detailed discussion of the so-called Whitehead Problem: recall that a group $G$ is said to be a Whitehead group if every extension of the group $\mathbb{Z}$ by $G$ splits, i.e., the group $\operatorname{Ext}(G, \mathbb{Z})=0$. Whitehead's problem had asked if every Whitehead group is necessarily free but Shelah showed in 1974 that the question is undecidable in the sense that it depends on the set-theoretic assumptions made. In some models of set theory all Whitehead groups are free but in other theories which are relatively consistent with ZFC, non-free Whitehead groups exist. Inevitably, discussions of large products of groups lead to set-theoretic issues concerning the existence of so-called $\omega$-measurable cardinals. A detailed discussion of these is not appropriate for this type of paper and so we 
restrict ourselves to the naive approach which considers these cardinals as being extraordinarily large. Further details may be found in the discussion on slender modules in [6, Chapter III] and in the section on the Axiom of Constructibility [6, Chapter VI].

First we consider the situation in relation to the group $P$. Recall that if $A$ is any subgroup of $P$, then adopting standard terminology from the theory of vector spaces, the annihilator $A^{\perp}$ of $A$, is given by $A^{\perp}=\{f \in \operatorname{Hom}(P, \mathbb{Z}): f(a)=0$ for all $a \in A\}$ and the second annihilator $A^{\perp \perp}$ of $A$, i.e., the subgroup of $P$ given by $\{x \in P$ : $f(x)=0$ for all homomorphisms $\left.f \in A^{\perp}\right\}$ is always a summand of $P$ - see, for example, [6, Chapter IX, Proposition 1.3]. However, if $\phi$ is an arbitrary surjection : $P \rightarrow P$ and $A=\operatorname{Ker} \phi$, then the group $P / A \cong P$ is certainly torsionless (it is even reflexive) and so it follows from [6, Chapter IX, Lemma 1.1] that $A=A^{\perp \perp}$. Thus Ker $\phi$ is a summand of $P$, and since $\phi$ was an arbitrary surjection, we have that $P$ is (sKer)-direct and hence R-Hopfian by Theorem 2.6 .

The situation becomes more complicated when we move to the higher Baer-Specker groups $P_{\kappa}$ with $\kappa>\aleph_{0}$. However, it is possible to use the argument above in another, more general, situation. If we make the set-theoretic assumptions that every Whitehead group of cardinality $\leq \kappa$ is free and that $\kappa$ is not too large in the technical sense that it is not $\omega$-measurable, then, as above, we can deduce that the subgroup $A$ is a summand using [6, Chapter IX, Theorem $1.5]$ and thus it easily follows that:

Theorem 4.1. (i) The Baer-Specker group $P$ is R-Hopfian;

(ii) if $\kappa$ is non- $\omega$-measurable and if every Whitehead group of cardinality $\leq \kappa$ is free, then $P_{\kappa}$ is $R$-Hopfian.

Our next result shows that if we make even stronger set-theoretical assumptions then the groups $P_{\kappa}$ are always R-Hopfian. We have outlined below an algebraic approach using standard results in [6], but it is possible to deduce the result by using the more technical approach due originally to Scott which shows that $\omega$-measurable cardinals cannot exist under the Axiom of Constructibility $(\mathrm{V}=\mathrm{L})$.

Theorem 4.2. If $(V=L)$, then the group $P_{\kappa}$ is $R$-Hopfian for every infinite cardinal $\kappa$.

Proof. It follows from [6, Chapter IX, Corollary 1.6] that for all infinite cardinals $\kappa$, a subgroup $A$ of $P_{\kappa}$ is a direct summand of $P_{\kappa}$ 
if, and only if, the quotient $P_{\kappa} / A$ is a product. In particular, if $\phi: P_{\kappa} \rightarrow P_{\kappa}$ is a surjection and $A=\operatorname{Ker} \phi$, then $\operatorname{Ker} \phi$ is a summand of $P_{\kappa}$. Hence the group $P_{\kappa}$ is (sKer)-direct and thus it is R-Hopfian by Theorem 2.6.

Our next result is based upon Example 1.7 in [6, Chapter IX].

Theorem 4.3. If there exists a non-free Whitehead group $B$ of non$\omega$-measurable cardinality $\kappa$, then the group $P_{\lambda}$ is not R-Hopfian for any cardinal $\lambda \geq \kappa$.

Proof. Choose a free resolution $0 \rightarrow G \rightarrow F \rightarrow B \rightarrow 0$ in which both $G$ and $F$ are free of rank $\kappa$ and apply the the functor $\operatorname{Hom}(-, \mathbb{Z})$ to get a short exact sequence

$$
0 \rightarrow B^{*} \rightarrow F^{*} \stackrel{\eta}{\rightarrow} G^{*} \rightarrow 0 \quad(*) .
$$

Note that $G^{*} \cong F^{*} \cong P_{\kappa}$ and fix an isomorphism $j: G^{*} \rightarrow F^{*}$. Define $\phi: F^{*} \rightarrow F^{*}$ by $\phi=j \eta$. Then Ker $\phi=\operatorname{Ker} \eta$ since $j$ is monic, so $\operatorname{Ker} \phi=B^{*}$. Note also that $\operatorname{Im} \phi=j \eta\left(F^{*}\right)=j\left(G^{*}\right)=F^{*}$, so that $\phi$ is onto. Claim that $B^{*}$ is not a summand of $F^{*}$; it follows from Theorem 2.6 that this will suffice to show that $F^{*} \cong P_{\kappa}$ is not R-Hopfian. Since a direct summand of an R-Hopfian group is again R-Hopfian (Proposition 2.3), this will ensure that $P_{\lambda}$ is not R-Hopfian for any $\lambda \geq \kappa$.

If $B^{*}$ were a summand of $F^{*}$, then the sequence $(*)$ would be splitting exact, giving that $G^{*}$ is a summand of $F^{*}$. Taking second duals and using the fact that products of $\mathbb{Z}$ over non- $\omega$-measurable indexing sets are reflexive, this would lead to the conclusion that the resolution $0 \rightarrow G \rightarrow F \rightarrow B \rightarrow 0$ splits, hence $B$ is free contradiction.

Since the existence of a non-free Whitehead group of cardinality $\aleph_{1}$ can be established under the set-theoretic assumption (MA + $\neg \mathrm{CH}$ ), our last result shows that Theorem 4.2 cannot be proved in $\mathrm{ZFC}$, and that it is independent of ZFC whether every higher Baer-Specker group $P_{\kappa}\left(\kappa \geq \aleph_{1}\right)$ is R-Hopfian.

\section{REFERENCES}

[1] R. Baer, Groups without Proper Isomorphic Quotient Groups, Bull. Amer. Math. Soc. 50 (1944) 267 - 278.

[2] G. Baumslag, Hopficity and Abelian Groups, in Topics in Abelian Groups, Scott Foresman, Chicago, Illinois (1963) 331-335. 
[3] R.A. Beaumont and R.S. Pierce, Isomorphic Direct Summands of Abelian Groups, Math. Ann. 153 (1964) 21-37.

[4] E. Coleman, The Baer-Specker Group, Bulletin Irish Math. Soc, 40 (1998) 9-23.

[5] A.L.S. Corner, Three Examples on Hopficity in Torsion-Free Abelian Groups, Acta Math. Acad. Sci. Hungar. 16 (1965) 303-310.

[6] P. Eklof and A. Mekler, Almost Free Modules, Set-theoretic Methods, NorthHolland, 2002.

[7] L. Fuchs, Infinite Abelian Groups, Vol.I, Academic Press 1970.

[8] L. Fuchs, Infinite Abelian Groups, Vol.II, Academic Press 1973.

[9] B. Goldsmith and K. Gong, Algebraic entropies, Hopficity and co-Hopficity of direct sums of Abelian Groups, Topol. Algebra Appl 3 (2015) 75 - 85.

[10] B. Goldsmith and K. Gong, A Note on Hopfian and co-Hopfian Abelian Groups, in Groups and Model Theory, Contemp. Math. 576 (2012) Amer Math. Soc., Providence R.I. $124-136$.

[11] B. Goldsmith and K. Gong, R-Hopfian and L-co-Hopfian Abelian groups, in Groups, Modules and Model Theory - Surveys and Recent Developments, (editors: M. Droste, L. Fuchs, B. Goldsmith and L. Strüngmann), Springer International Publishing 2017, pp 333 - 349.

[12] B. Goldsmith and P. Vámos, The Hopfian Exponent of an Abelian Group, Period. Math. Hung. 69 (2014) 21-31.

[13] R. Hirshon, On Hopfian groups, Pacific J. Math. 32 (1970) 753-766.

[14] G. Lee, S.T. Rizvi and C.S. Roman, Rickart Modules, Comm. Algebra, 38 (2010) 4005-4027.

[15] G. Lee, S.T. Rizvi and C.S. Roman, Dual Rickart Modules, Comm. Algebra, 39 (2011) 4036-4058.

[16] P.M. Neumann, Pathology in the Representation Theory of Infinite Soluble Groups, in Proc. 'Groups-Korea 1988' Lecture Notes in Mathematics 1398 (Eds A.C. Kim and B.H. Neumann) 124-139.

[17] R.S. Pierce, Homomorphisms of Primary Abelian Groups, in Topics in Abelian Groups, Scott Foresman, Chicago, Illinois (1963), 215-310.

[18] K.M. Rangaswamy, Abelian Groups with Endomorphic Images of Special Types, J. Algebra 6 (1967) 271-280.

Brendan Goldsmith is President Emeritus at Dublin Institute of Technology and a former president of the IMS. His research interests are in algebra.

School of Mathematical Sciences,, The Clock Tower, CT032, Dublin Institute of Technology,, Grangegorman Lower,, Dublin D07H6K8, IRELAND.

E-mail address: brendan.goldsmith@dit.ie 